\title{
Caractérisation et stabilisation de quelques sols gonflants d'Algérie
}

\section{A. HACHICHI}

Chargé de cours Institut de Génie Civi] Université des Sciences et de la Technologie BP 1505

El M'naouar Oran, Algérie

\section{J.-M. FLEUREAU}

Professeur Laboratoire de Mécanique des Sols,

Structures et Matériaux

CNRS UMR 8579

et École Centrale de Paris

Grande Voie des Vignes 92295 Châtenay-Malabry,
L'article présente un ensemble de mesures réalisées sur des argiles gonflantes provenant de cinq sites différents dans plusieurs régions d'Algérie, sur lesquelles on avait observé des désordres dans les constructions.

Dans la première partie, les matériaux ont été

caractérisés du point de vue de leur minéralogie, de leurs dimensions et de leur plasticité, ce qui a permis d'établir un classement des potentiels de gonflement de chacun ; les résultats très cohérents fournis par les cinq méthodes utilisées ont été confirmés par des mesures directes de gonflement libre et de pression de gonflement. Dans la deuxième partie, l'étude porte sur l'influence de différentes solutions salines sur le gonflement; les sels de calcium et d'ammonium, à des concentrations molaires supérieures ou égales à $0,5 \mathrm{M}$, en particulier, peuvent conduire à des réductions très importantes du gonflement, pouvant atteindre $80 \%$ avec certaines argiles. D'une manière générale, I'utilisation de tels traitements nécessite une étude spécifique pour chaque cas envisagé,

Mots clés : argiles gonflantes, gonflement, pression de gonflement, stabilisation chimique, Algérie.

\section{Characterization and stabilization of a few expansive soils from Algeria}

The paper presents a series of measurements on expansive clays taken from five different sites in several regions of Algeria, where disorders had been observed in buildings. In the first part, the mineralogy of the materials, their dimensions and their plasticity parameters are determined, which leads to a filing of their swelling potentials; the very consistent results derived from the five methods used were confirmed by direct measurements of free swells and swelling pressures.

In the second part, the influence of several salt solutions on free swell was studied : calcium and ammonium salts, at concentrations equal to or larger than $0,5 \mathrm{M}$, in particular, may lead to very large decreases in free swell, that can reach $80 \%$ for some clays. Generally speaking, the use of such treatments requires a specific study for each soil considered.
}

Key words : expansive soils. swelling, swelling pressure. chemical treatment, Algeria. 


\section{NOTATIONS}

\begin{tabular}{|c|c|}
\hline & \\
\hline$w_{1}$ & : teneur en eau initiale $(\%)$ \\
\hline$w_{\text {nat }}$ & : teneur en eau naturelle (\%). \\
\hline$w_{L}$ & : limite de liquidité. \\
\hline$W_{p}$ & : limite de plasticité. \\
\hline & : indice de plasticité. \\
\hline$v_{S L}$ & : limite de retrait $(\%)$. \\
\hline$W_{\text {mes }}$ & $\begin{array}{l}\text { : teneur en eau au moment de la mesure d'un } \\
\text { paramètre }(\%) \text {. }\end{array}$ \\
\hline & : indice des vides. \\
\hline & : degré de saturation. \\
\hline$\gamma_{s} / \gamma_{w}$ & : densité des grains solides. \\
\hline 80 & $\begin{array}{l}\text { pourcentage de grains de diamètre inférieur } \\
\text { à } 80 \mu \mathrm{m} \text {. }\end{array}$ \\
\hline $\mathrm{P}_{2}$ & $\begin{array}{l}\text { : pourcentage de grains de diamètre inférieur } \\
\text { à } 2 \mu \mathrm{m} \text {. }\end{array}$ \\
\hline$D_{60}$ & $\begin{array}{l}\text { : diamètre correspondant à } 60 \% \text { de passants } \\
(\mu \mathrm{m}) .\end{array}$ \\
\hline$A_{e}$ & : activité $\left[=I_{p} / P_{2}\right]$ \\
\hline$A_{\text {cor }}$ & : activité corrigée $\left[=I_{p} /\left(\mathrm{P}_{2}-10\right)\right]$ : \\
\hline$V_{B}$ & : valeur de bleu. \\
\hline$S_{p}$ & : surface spécifique totale $\left(\mathrm{m}^{2} / \mathrm{g}\right)$. \\
\hline G & : gonflement libre (en \%). \\
\hline & : pression de gonflement $(\mathrm{kPa})$ \\
\hline$t_{100}$ & $\begin{array}{l}\text { : temps correspondant à la fin du gonflement } \\
\text { primaire (mn). }\end{array}$ \\
\hline$u_{w}$ & : pression interstitielle négative $(\mathrm{kPa})$. \\
\hline$u_{w 51}$ & : pression négative de retrait. \\
\hline Awdént & : pression négative de désaturation. \\
\hline
\end{tabular}

\section{1}

\section{Introduction}

Les sols gonflants, relativement répandus en Algérie, occasionnent des désordres importants aux ouvrages qu'ils supportent (Fig. 1). On a recensé de nombreux cas de pathologies d'ouvrages (raffinerie, habitations, hôpital, etc.) dues à ce type de sols dans différentes régions, ce qui nous a amené à nous intéresser à quelques-uns d'entre eux afin d'étudier leurs caractéristiques, leur comportement lors de l'humidification et une méthode permettant de réduire le gonflement.

Le phénomène d'humidification d'une argile séchée peut être étudié de façon détaillée en soumettant des échantillons de sol à des pressions interstitielles négatives (ou succions) connues et en mesurant ensuite les changements d'état du matériau une fois l'équilibre de pression atteint. De nombreuses méthodes ont été décrites dans la littérature, qui permettent de réaliser ces essais (par exemple, Biarez et al., 1988). La figure 2 représente l'état final d'échantillons de montmorillonite- $\mathrm{Ca}\left(\mathrm{w}_{1}=170, \mathrm{I}_{\mathrm{p}}=110\right)$ sur un chemin de drainagehumidification. Le matériau, préparé initialement sous forme d'une pâte très liquide ( $\left.w_{1}=1,5 w_{1}\right)$ est ensuite consolidé dans un cedomètre sous une contrainte verticale de $100 \mathrm{kPa}$. Après sortie du moule, sa pression négative s'établit à une valeur de l'ordre de $50 \mathrm{kPa}$, insuffisante pour désaturer l'argile. Les résultats sont tracés sur cinq graphiques qui se correspondent, en fonction de la pression négative imposée et de la teneur en eau (mesurée) du sol. On constate que :

- dans le plan [-u, e], l'argile suit d'abord un chemin surconsolidé sans se désaturer; lorsque celui-ci rejoint le chemin normalement consolidé (défini par des essais sur la pâte d'argile), le matériau commence à se désaturer fortement et son indice des vides devient pratiquement constant jusqu'à atteindre un état complètement sec. La pression négative au début du palier, égale à $2500 \mathrm{kPa}$ environ, appelée pression négative de retrait et notée $\left(-u_{w}\right)_{\mathrm{SL}}$, correspond à un profond changement de comportement mécanique du sol. Lorsque la pression négative diminue, l'argile commence par se rehumidifier à volume sensiblement constant jusqu'à $\left(-u_{w}\right)_{\mathrm{S}}$, puis son volume augmente tandis qu'elle finit de se saturer. Par conséquent, en draínage comme en humidification, l'essentiel des variations de volume se produit dans le domaine saturé ou quasi saturé. Si l'on fait subir au matériau un nouveau cycle de séchage et d'humidification, le chemin suivi est quasiment réversible et confondu avec le chemin d'humidification du premier cycle (Fleureau et al., 1998) ;

- dans le plan [-u, $\mathrm{S}_{r}$, les chemins de séchage et d'humidification (complétés au-delà de $10^{5} \mathrm{kPa}$ en tenant compte des nombreux résultats existants pour d'autres argiles) sont pratiquement superposés. Les pressions négatives de désaturation (-u $\left.u_{\text {wajar }}\right)$ et de resa-

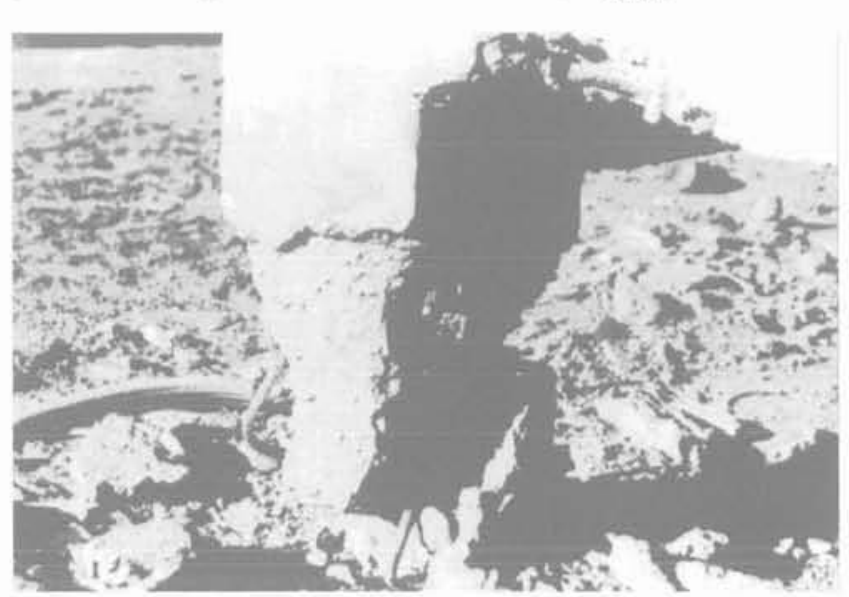

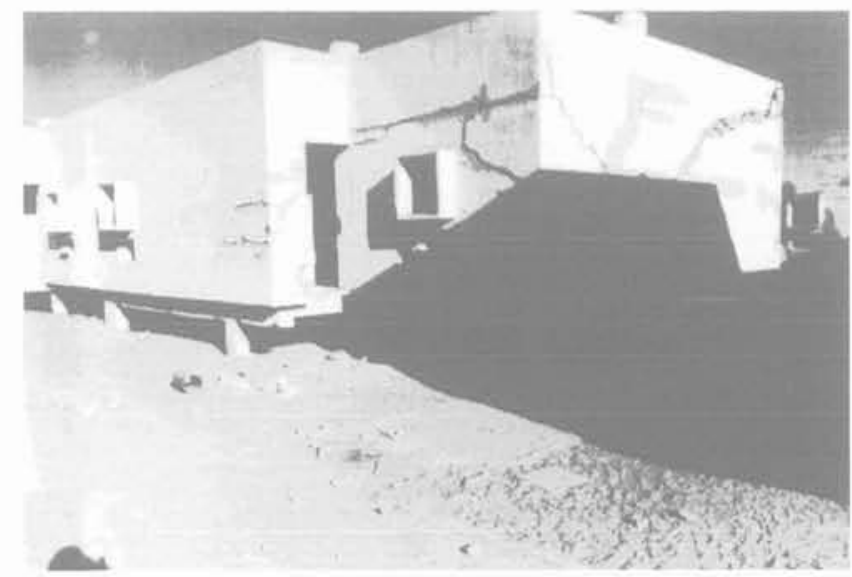

FIG.1 Exemples de désordres dans les constructions dus au gonflement des sols, observés à In Amenas (Algérie). Examples of disorders in buildings due to soil swelling observed in In Amenas (Algeria). 

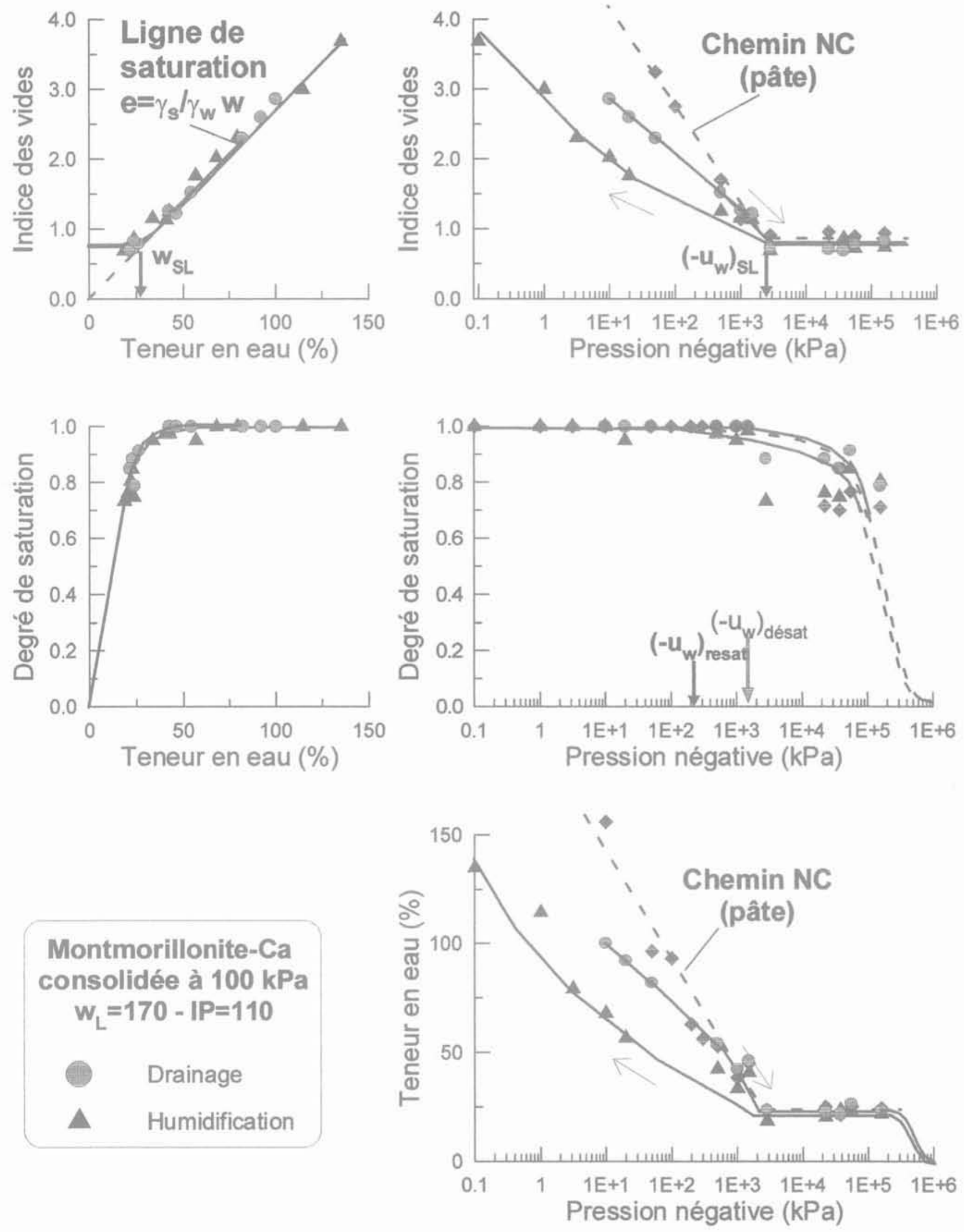

FG. 2. Chemins de drainage et d'humidification sur une argile gonflante préparée initialement sous forme de pâte saturée à une teneur en eau égale à 1,5 fois la limite de liquidité puis consolidée sous $100 \mathrm{kPa}$. Drying and wetting paths on an expansive clay first prepared as a slurry at an initial water content equal to 1.5 time the liquid limit, then consolidated under a $100 \mathrm{kPa}$ stress. 
turation $\left(-\mathrm{u}_{\text {wresppt }}\right)$ atteignent des valeurs très élevées dans ce materiau très plastique, respectivement de 1500 et $200 \mathrm{kPa}$;

- dans le plan [w, e], la limite de retrait correspond à la pression négative de retrait. Dans ce plan, pour presque toutes les argiles étudiées, la réversibilité des chemins est parfaite, de même que dans le plan [w, S] (Fleureau et al., 1993). Par conséquent, quelle que soit la teneur en eau initiale d'un sol, lorsque celle-ci est inférieure ou égale à la limite de retrait, il subira le même gonflement libre :

- enfin le plan $\left[-u_{w}, w\right]$ est pratiquement superposé au plan $\left[-u_{w}\right.$ e] jusqu'a la pression négative de $100 \mathrm{MPa}$, pour laquelle la désaturation de l'argile devient significative.

Dans la pratique, deux grandeurs sont utilisées pour caractériser l'aptitude d'un sol au gonflement :

- le gonflement libre, correspondant à la variation relative de volume (en \%) d'un échantillon soumis à une surcharge nulle ou très faible (généralement le poids du piston dans un œdomètre) lorsqu'on le met en contact avec de l'eau à pression nulle. Le gonflement est constitué d'une composante " osmotique x due à la différence de concentration en sels de l'eau interstitielle et d'une composante "matricielle » régie par la pression interstitielle négative initiale de l'échantillon qui joue, dans la plupart des cas, un rôle majeur

- la pression de gonflement qui, dans un œdomètre, est définie comme la contrainte verticale dẻveloppée par un échantillon dans les mêmes conditions que pour la mesure de gonflement libre iorsque ses déformations verticale et latérale sont empêchées,

Naturellement, tous les essais intermédiaires entre ces deux extrêmes peuvent être envisacés, et permettent de tracer des courbes de variation des contraintes développées par un échantillon, pour des conditions initiales imposées, en fonction de ses déformations. Le gonflement libre et la pression de gonflement unidimensionnels sont fortement corrélés, mais correspondent à des chemins très différents, ce qui ne permet pas de les relier simplement.

Dans la plupart des méthodes, les conditions initiales sont, soit l'état naturel de l'échantillon non remanié lors de son prélèvement (mais celui-ci dépend des conditions climatiques, de la saison, etc.), soit un état fixé arbitrairement correspondant généralement aux conditions les plus sévères de sécheresse que le sol est susceptible de connaitre, c'est-à-dire souvent à une teneur en eau inférieure à la limite de retrait.

Notre étude a été réalisée à partir des données de cinq sites situés dans trois régions du pays, dont les sols sont à l'origine de fissurations de bâtiments, soulèvements de fonds de fouilles, etc.

Les sols ont été caractérisés par des analyses minéralogiques, pour identifier les minéraux susceptibles de provoquer des déformations volumiques importantes (notamment, la montmorillonite), et par des essais d'identification classiques (limites d'Atterberg, granutométrie, valeur de bleu, etc.). Ces essais ont été complétés par des mesures directes de gonflement et de pression de gonflement. Dans une seconde étape, l'étude a porté sur l'effet de différents sels, et de leur concentration, sur l'amplitude et la cinétique du gonflement, afin d'en déduire une méthode pratique de stabilisation du phénomène.

\section{Localisation et géologie des sites}

- Le site d'Abadla est situé au sud-ouest du pays ; il est formé de grès argileux très fins à stratification oblique. Les coupes de sondages ont révélé des argiles rouges et des marnes brunes et vertes.

- Le site d'Ouled-Khodeir, situé au sud-ouest du pays, est formé d'argiles brunes avec des traces de glissement.

- Le fossé de Mers El Kebir, qui est situé au nordouest du pays, est constitué par des marnes grises et vertes. Ce sont des formations sédimentaires marines, lagunaires vers le sommet, connues sous l'appellation des marnes bleues du Miocène supérieur. Ces marnes peuvent parfois dépasser 300 mètres d'épaisseur.

- Le site de Sidi Chami, situé au nord-ouest du pays, est constitué de deux couches superposées. La première couche est composée d'argile lacustre du quaternaire avec des passées sableuses et calcaires. La deuxième couche, sous-jacente à la première, est formée de marne plus ou moins sableuse du Pliocène.

- Enfin, le site d'In Amenas, situé au sud-est du pays, est formé d'argiles très raides, bariolées, du Trias.

\section{3}

\section{Analyse minéralogique}

Une analyse diffractométrique aux rayons $\mathrm{X}$ a été menée pour déterminer la minéralogie des minéraux, argileux ou non, constituant les sols étudiés.

Cette analyse a montré que l'argile de In Amenas était constituée de $40 \%$ de kaolinite, de $25 \%$ d'illite, de $35 \%$ d'interstratifiés illite-montmorillonite et de $23 \%$ de quartz.

L'argile d'Abadla est constituée d'illite, de kaolinite et de chlorite, ainsi que de quartz, de calcite et de fer.

L'argile de Ouled Khodeir est composée essentiellement de montmorillonite, d'illite, de kaolinite, de calcite, de quartz et de quelques débris de feldspaths.

L'arcile de Sidi Chami est formée de $20 \%$ de kaolinite, de $40 \%$ d'illite, de 35 à $40 \%$ d'interstratifiés illitemontmorillonite, de $12 \%$ de quartz et de 12 à $16 \%$ de calcite.

L'analyse diffractométrique n'a pas été faite sur l'argile de Mers El Kebir.

\section{4}

\section{Essais d'identification}

Des essais d'identification ont été effectués sur des échantillons intacts et remaniés, prélevés à différentes profondeurs. Les résultats des essais sont rassemblés dans le tableau I, où figurent les mesures suivantes:

- les limites d'Atterberg (limite de liquidité, $w_{1}$, et indice de plasticité, $\left.I_{p}\right)$ :

- la granulométrie, caractérisée par les pourcentages de grains plus petits que $80 \mu \mathrm{m}$ et $2 \mu \mathrm{m}$ (respectivement notés $\mathrm{P}_{80}$ et $\mathrm{P}_{2}$ ) et par le diamètre correspondant à $60 \%$ de passants (noté $\mathrm{D}_{60}$ );

$$
\text { - l'activité : } A_{c}=I_{p} / P_{2} \text {; }
$$


TABLEAUI Caractéristiques d'identification des matériaux étudiés.

\begin{tabular}{|c|c|c|c|c|c|c|c|c|c|c|c|}
\hline Matériau & Sondage & Ech. & $W_{L}$ & I & $\begin{array}{c}\left.\mathrm{P}_{(\%}<80 \mu \mathrm{m}\right) \\
\left(\%<{ }^{2}\right)\end{array}$ & $\begin{array}{c}\mathrm{P}_{2} \\
(\%<2 \mu \mathrm{m})\end{array}$ & $\begin{array}{l}\mathrm{D}_{6 \mathrm{~m}} \\
(\mu \mathrm{m})\end{array}$ & $A_{c}$ & $\mathrm{~V}_{\mathrm{B}}$ & $W_{\%}$ & $\begin{array}{c}W_{S+} \\
\%\end{array}$ \\
\hline \multirow[t]{3}{*}{ Abadla } & S1 & $\begin{array}{l}\mathrm{A} \\
\mathrm{B}\end{array}$ & $\begin{array}{l}40,2 \\
41,2\end{array}$ & $\begin{array}{l}17,1 \\
17,9\end{array}$ & $\begin{array}{l}93 \\
93\end{array}$ & $\begin{array}{l}29 \\
30\end{array}$ & $\begin{array}{l}7 \\
6\end{array}$ & $\begin{array}{l}0,6 \\
0,6\end{array}$ & 7,5 & $\begin{array}{l}40,2 \\
41,2\end{array}$ & $\begin{array}{l}14,8 \\
15,6\end{array}$ \\
\hline & S2 & C & 30,8 & 15,5 & 84 & 13 & 21 & 1,2 & & 30.8 & 11,3 \\
\hline & S3 & D & 41,4 & 21,1 & 92 & 21 & & 1 & & 41,4 & 14.7 \\
\hline \multirow[t]{3}{*}{ Ouled Khodeir } & S1 & $\begin{array}{l}\mathrm{A} \\
\mathrm{B}\end{array}$ & $\begin{array}{l}50 \\
50,9\end{array}$ & $\begin{array}{l}22,3 \\
21,8\end{array}$ & $\begin{array}{l}95 \\
95\end{array}$ & $\begin{array}{l}42 \\
41\end{array}$ & $\begin{array}{l}3 \\
3\end{array}$ & $\begin{array}{l}0,6 \\
0,5\end{array}$ & 7,2 & $\begin{array}{l}50 \\
50,9\end{array}$ & $\begin{array}{l}16,3 \\
15,5\end{array}$ \\
\hline & $\$ 1$ & $\begin{array}{l}1 \\
2 \\
3 \\
4\end{array}$ & $\begin{array}{c}110 \\
107 \\
86,4 \\
75\end{array}$ & $\begin{array}{l}78,9 \\
73 \\
61,1 \\
46,8\end{array}$ & & $\begin{array}{l}25 \\
28 \\
20 \\
35\end{array}$ & & $\begin{array}{l}3,2 \\
2,7 \\
3,1 \\
1,8\end{array}$ & 8,1 & $\begin{array}{l}12,3 \\
39,9 \\
25,5 \\
26,3\end{array}$ & \\
\hline & $\$ 2$ & $\begin{array}{l}1 \\
2 \\
3 \\
4 \\
5\end{array}$ & $\begin{array}{l}30 \\
42,1 \\
52 \\
91 \\
82,5\end{array}$ & $\begin{array}{l}15,6 \\
24,3 \\
30,2 \\
62 \\
57,6\end{array}$ & & $\begin{array}{l}16 \\
14 \\
20 \\
42 \\
40\end{array}$ & & $\begin{array}{l}1 \\
1,7 \\
1,5 \\
1,4\end{array}$ & 4,8 & $\begin{array}{l}14,6 \\
21,3 \\
26,9 \\
26\end{array}$ & \\
\hline \multirow[t]{3}{*}{ Sidi Chami } & S3 & $\begin{array}{l}1 \\
2 \\
3 \\
4\end{array}$ & $\begin{array}{r}28,8 \\
18,5 \\
76,7 \\
105\end{array}$ & $\begin{array}{r}13,3 \\
6,6 \\
51,5 \\
71\end{array}$ & & $\begin{array}{l}6 \\
7 \\
48 \\
60\end{array}$ & & $\begin{array}{l}2,2 \\
0,9 \\
1,1 \\
1,2\end{array}$ & 8,3 & $\begin{array}{l}14,6 \\
21,3 \\
26,9 \\
26\end{array}$ & \\
\hline & 54 & $\begin{array}{l}1 \\
2 \\
3 \\
4\end{array}$ & $\begin{array}{l}37 \\
89,2 \\
91 \\
61\end{array}$ & $\begin{array}{l}20,3 \\
57,7 \\
62,8 \\
40,8\end{array}$ & & $\begin{array}{l}10 \\
48 \\
46 \\
43\end{array}$ & & $\begin{array}{l}2 \\
1,2 \\
1,4 \\
1\end{array}$ & 5,3 & $\begin{array}{l}23,5 \\
34,2 \\
38,8 \\
24,6\end{array}$ & \\
\hline & $\$ 6$ & $\begin{array}{l}1 \\
2 \\
3 \\
4 \\
5 \\
6\end{array}$ & $\begin{array}{l}81,5 \\
50 \\
68,6 \\
82,9 \\
69,8 \\
73,5\end{array}$ & $\begin{array}{l}62,5 \\
30,2 \\
48,5 \\
62 \\
44,2 \\
50,3\end{array}$ & & $\begin{array}{l}34 \\
27 \\
46 \\
30 \\
55 \\
52\end{array}$ & & $\begin{array}{l}1,8 \\
1,1 \\
1,1 \\
1,3 \\
0,8 \\
1\end{array}$ & & & \\
\hline \multirow[t]{8}{*}{ Mers El Kebir } & S1 & 1 & 57,5 & 28,9 & & 42 & & 0,7 & & 19,3 & 19,5 \\
\hline & $\$ 2$ & 2 & 47,5 & 24,7 & & 42 & & 0,6 & & 16,8 & 17,3 \\
\hline & S3 & 3 & 57 & 34 & & 38 & & 0,9 & & 18,8 & 18,2 \\
\hline & S4 & $\begin{array}{l}4 \\
5\end{array}$ & $\begin{array}{l}45 \\
52,5\end{array}$ & $\begin{array}{l}22,8 \\
27,3\end{array}$ & & $\begin{array}{l}39 \\
42\end{array}$ & & $\begin{array}{l}0,6 \\
0,7\end{array}$ & & $\begin{array}{l}14,9 \\
16,3\end{array}$ & $\begin{array}{l}15,9 \\
15,4\end{array}$ \\
\hline & S5 & $\begin{array}{l}6 \\
7\end{array}$ & $\begin{array}{l}64 \\
51,5\end{array}$ & $\begin{array}{l}38,5 \\
26,6\end{array}$ & & $\begin{array}{l}40 \\
35 \\
\end{array}$ & & $\begin{array}{l}1 \\
0,6\end{array}$ & & $\begin{array}{l}22,9 \\
14,9\end{array}$ & $\begin{array}{l}21,8 \\
17\end{array}$ \\
\hline & S6 & $\begin{array}{l}8 \\
9\end{array}$ & $\begin{array}{l}51 \\
51\end{array}$ & $\begin{array}{l}24,3 \\
27,2\end{array}$ & & $\begin{array}{l}42 \\
43\end{array}$ & & $\begin{array}{l}0.6 \\
0,6\end{array}$ & & $\begin{array}{l}18,8 \\
16,5\end{array}$ & $\begin{array}{l}20,5 \\
16,2\end{array}$ \\
\hline & S7 & $\begin{array}{l}10 \\
11\end{array}$ & $\begin{array}{l}55,5 \\
48,5\end{array}$ & $\begin{array}{l}29,7 \\
26,6\end{array}$ & & $\begin{array}{l}45 \\
34\end{array}$ & & $\begin{array}{l}0,7 \\
0,8\end{array}$ & & $\begin{array}{l}17,2 \\
15,5\end{array}$ & $\begin{array}{l}18,9 \\
16,2\end{array}$ \\
\hline & S8 & $\begin{array}{l}\mathrm{A} \\
\mathrm{B} \\
\mathrm{C}\end{array}$ & $\begin{array}{l}52 \\
51,7 \\
50,5\end{array}$ & 27,3 & & 32 & & 0,9 & 19,9 & $\begin{array}{l}12,8 \\
14,6 \\
11,2\end{array}$ & 14,7 \\
\hline In Amenas & S1 & $A$ & 62,3 & 40,6 & 95 & 55 & 2 & 0,8 & 14,7 & 22,5 & 12 \\
\hline
\end{tabular}

- la valeur de bleu, $V_{\mathrm{B}}$;

- la teneur en eau naturelle ;

- la limite de retrait effective, déterminée par la méthode de mesure du retrait linéaire, selon la norme XP P94-060-2.

La limite de liquidité des sols varie très largement d'un échantillon à l'autre, entre 30 et $110 \%$ et l'indice de plasticité est compris entre 15 et $79 \%$. Parmi les cinq sols étudiés, celui de Sidi Chami présente la limite de liquidité la plus élevée, soit $110 \%$. En utilisant l'abaque de plasticité de Casagrande (Fig. 3), on remarque que les argiles d'Abadla et d'Ouled Khodeir se classent parmi les argiles moyennement plastiques, et celles de Mers El Kebir, Sidi Chami et In Amenas, dans les argiles fortement plastiques.

Les valeurs de surface spécifique totale $\left(S_{p}\right)$, déduites de l'essai au bleu de méthylène par la formule (Tran Ngoc Lan, 1977): 


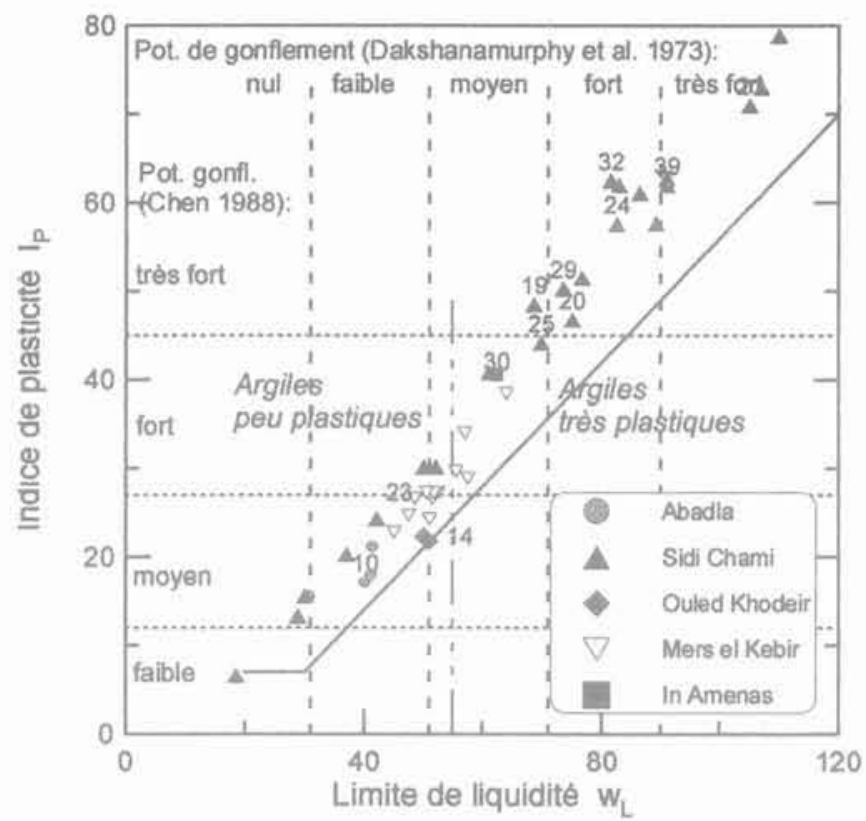

Classification des potentiels de gonflement de Dakshanamurphy \& Raman (1973) et Chen (1988)

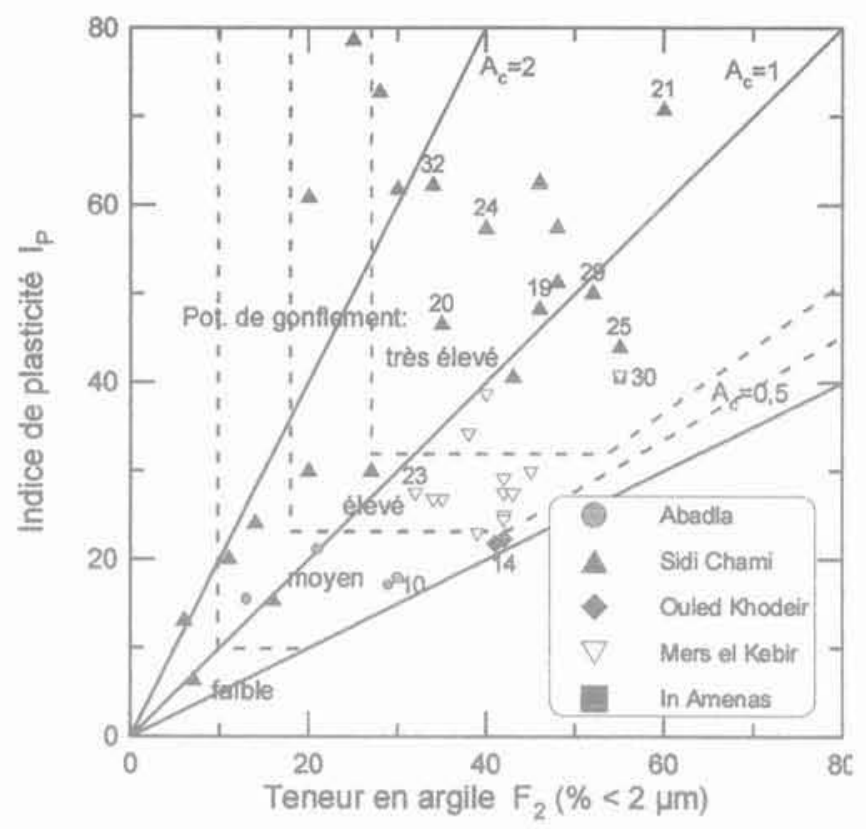

Classification des potentiels de gonflement de Williams \& Donaldson (1980)

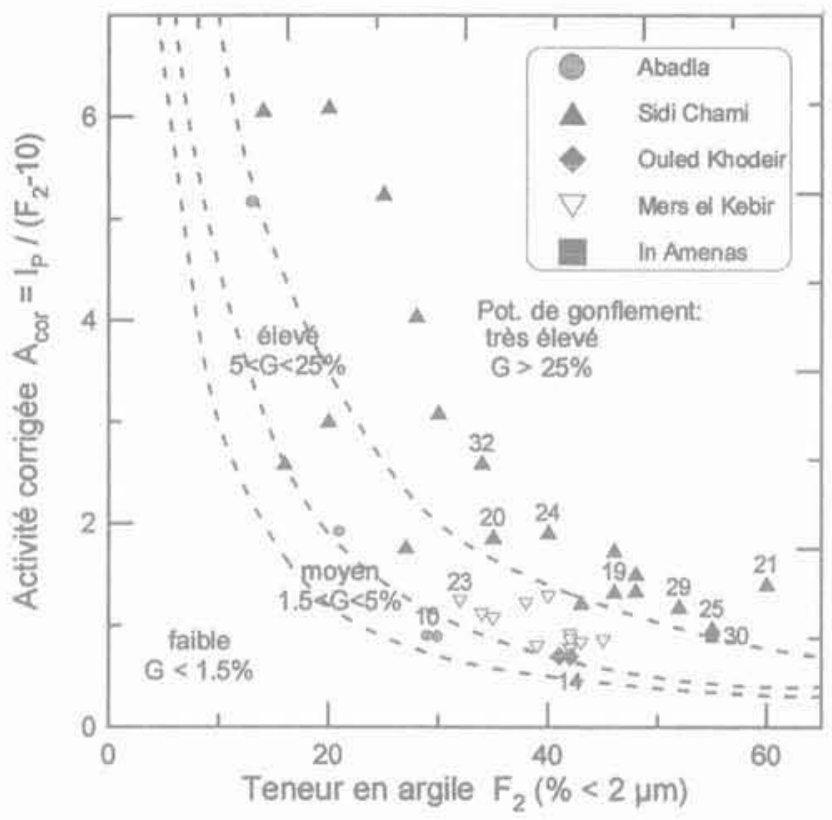

Classification des potentiels de gonflement de Seed et al. (1962)

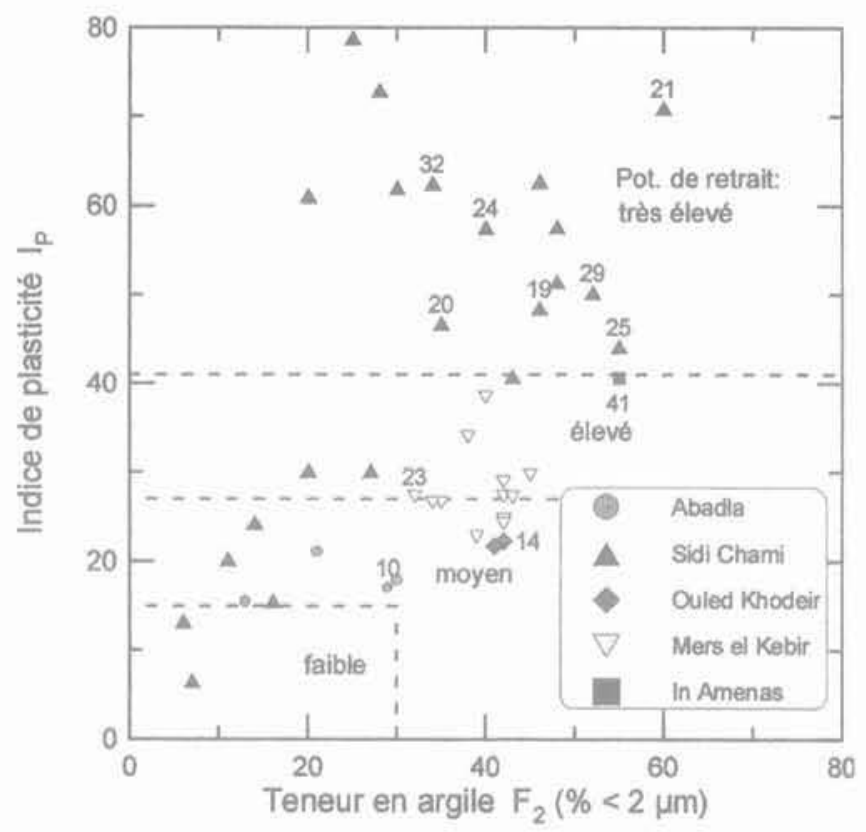

Classification des potentiels de retrait du Building Research Establishment (1980)

FIG. 3 Classification du potentiel de gonflement ou de retrait des sols en fonction des paramètres d'identification (les gonflements libres mesurés sont indiqués en étiquettes des données).

Filing of swelling or shrinkage potentials of soils as a function of identification parameters (measured free swells are indicated as data labels).

$$
\mathrm{S}_{\mathrm{p}}\left[\mathrm{m}^{2} / \mathrm{g}\right]=21 \mathrm{~V}_{\mathrm{B}} \times \mathrm{P}_{2}
$$

$\mathrm{m}^{2} / \mathrm{g}$ pour l'argile d'In Amenas et $400 \mathrm{~m}^{2} / \mathrm{g}$ pour l'argile de Mers El Kebir. Ces valeurs indiquent la présence de forts pourcentages de montmorillonite dans les deux dernières argiles $\left(300<\mathrm{S}_{\mathrm{p}}<800\right)$ et de pourcentages non négligeables dans les autres matériaux. 


\section{Caractérisation du potentiel de gonflement}

De nombreuses méthodes ont été proposées dans la littérature pour évaluer a priori le potentiel de gonflement d'un sol à partir de la mesure des paramètres de plasticité et de granulométrie - par exemple Seed et al., 1962, Komornik \& David, 1969, Vijayvergiya \& Ghazzaly 1973, Dakshanamurphy \& Raman, 1973 (in Meisina, 1996), Brackley 1975, Williams \& Donaldson, 1980, le Building Research Establishment [BRE], 1980 (in Taylor \& Smith, 1980), Chen, 1988.

Seed et al. (1962) définissent le potentiel de gonflement comme la déformation volumique d'un échantillon compacté à la densité sèche maximale (selon la norme AASHO), confiné latéralement, soumis à une charge verticale de $0,7 \mathrm{kPa}$ et en contact avec l'eau libre.

Nous avons reporté les points expérimentaux obtenus sur la figure 3 , dans les axes correspondant à quelques-unes de ces classifications :

- la limite de liquidité $\mathrm{w}_{1}$ et l'indice de plasticité $\mathrm{I}_{\mathrm{p}}$ pour les méthodes de Dakshanamurphy \& Raman et Chen;

- la teneur en éléments plus petits que $2 \mu \mathrm{m}\left(\mathrm{F}_{2}\right)$ et l'indice de plasticité $\left(\mathrm{I}_{\mathrm{p}}\right)$ pour les méthodes de Williams \& Donaldson et du BRE;

- la teneur en argile $\mathrm{F}_{2}$ et l'activité corrigée, définie par $A_{c o r}=I_{p} /\left(F_{2}-10\right)$ pour la méthode de Seed et al. (1962).

Pour ces auteurs, un potentiel de gonflement très élevé correspond à un gonflement libre (exprimé en pourcentage) supérieur à $25 \%$, un potentiel élevé, à un gonflement compris entre 5 et $20 \%$, un potentiel moyen, à un gonflement compris entre 1,5 et $5 \%$ et un potentiel faible, à un gonflement inférieur à 1,5\%. Pour Komarnik et David (1969), les pressions de gonflement correspondantes sont, respectivement, supérieures à $300 \mathrm{kPa}$ (potentiel très élevé), comprises entre 200 et $300 \mathrm{kPa}$ (élevé), entre 100 et $200 \mathrm{kPa}$ (moyen) et inférieures à $100 \mathrm{kPa}$ (faible).

On constate un bon accord général entre ces classifications, à l'exception de celle de Williams \& Donaldson qui conduit à des potentiels de gonflement plus faibles que les autres, mais la hiérarchie est respectée. Notons qu'il s'agit, dans le cas de la méthode du BRE d'une estimation du potentiel de retrait et non de gonflement, mais les deux notions sont étroitement liées, comme nous l'avons précisé dans l'introduction. Après avoir comparé les estimations de divers auteurs et les mesures faites sur une centaine d'échantillons de plusieurs sols différents, Meisina (1993, 1996) conclut que c'est la méthode de Seed et al, qui se rapproche le plus de la réalité, tandis que les autres méthodes ont tendance à surestimer le potentiel de gonflement.

Le classement des différents matériaux par ordre de potentiels de gonflement croissants est le suivant (tableau II) :

- argile de Sidi Chami (sondages S2/S3) ;

- argiles d'Abadla et Ouled Khodeir ;

- argile de Mers El Kebir ;

- argiles de Sidi Chami (sondages S1/S4/ S6) et In Amenas.

Ce classement ne correspond pas à celui des valeurs de bleu (ou des surfaces spécifiques) pour lequel les valeurs les plus fortes étaient obtenues pour les argiles de In Amenas et Mers El Kebir, tandis que des valeurs plus faibles avaient été mesurées sur les trois autres sols, notamment Sidi Chami qui présente ici le potentiel le plus élevé. Ceci peut être dû au petit nombre de mesures de valeurs de bleu réalisées.

D'autres auteurs proposent des relations entre le gonflement ou la pression de gonflement et des paramètres de nature $\left(w_{1}, I_{p}\right)$ ou d'état (teneur en eau, densité sèche, pression interstitielle négative) du sol. Il s'agit, dans la plupart des cas, de corrélations empiriques déduites d'un nombre réduit d'essais. Une très bonne analyse de la validité de certaines de ces expressions est faite dans la thèse de Guiras-Skandaji (1996).

\section{6}

\section{Mesure du gonflement et de la pression de gonflement à l'œdomètre}

\section{1}

\section{Méthodes de mesure}

Comme nous l'avons vu, le gonflement libre est mesuré à l'œdomètre sur un échantillon soumis au seul

TABLEAU II Comparaison des prévisions de potentiels de gonflement pour quelques méthodes proposées dans la littérature.

\begin{tabular}{|c|c|c|c|c|c|}
\hline \multirow[b]{2}{*}{ Matériau } & \multicolumn{4}{|c|}{ Potentiel de gonflement } & \multirow{2}{*}{$\begin{array}{c}\text { Potentiel de retraft } \\
\text { Classification } \\
\text { du B.R.E. (1980) }\end{array}$} \\
\hline & $\begin{array}{c}\text { Classification } \\
\text { de Dakshanamurphy } \\
\text { et Raman (1973) }\end{array}$ & $\begin{array}{l}\text { Classification } \\
\text { de Chen (1988) }\end{array}$ & $\begin{array}{c}\text { Classification } \\
\text { de Williams } \\
\text { \& Donaldson (1980) }\end{array}$ & $\begin{array}{c}\text { Classiffication } \\
\text { de Seed et al. (1962) }\end{array}$ & \\
\hline Abadia & Moyen & Moyen & Faible & Moyen & Moyen \\
\hline $\begin{array}{l}\text { Sidi Chami } \\
\text { S1/S4/S6 }\end{array}$ & Très fort & Fort & Fort & Très fort & Très fort \\
\hline $\begin{array}{l}\text { Sidi Chami } \\
\text { S2/S3 }\end{array}$ & Faible à moyen & Faible à moyen & Faible à moyen & Fort & Faible à moyen \\
\hline Ouled Khodeir & Moyen à fort & Moyen & Faible à moyen & Moyen à fort & Moyen \\
\hline Mers El Kebir & Fort & Moyen à fort & Faible à moyen & Fort & Fort \\
\hline In Amenas & Très fort & Fort & Moyen & Très fort & Très fort à fort \\
\hline
\end{tabular}


poids du piston, mis au contact d'un réservoir d'eau à charge nulle. La variation de hauteur du piston est mesurée en fonction du temps (Fig. 4 à 6) jusqu'à ce qu'elle se stabilise; la valeur finale permet de calculer la variation relative de volume de l'échantillon, notée $\mathrm{G}$, que l'on exprime souvent en pourcentage. La courbe de gonflement présente deux parties que l'on peut analyser, par analogie avec le phénomène de consolidation, comme des phases de gonflement primaire et secondaire.

Il existe une multitude de méthodes pour mesurer la pression de gonflement à l'cedomètre. Les méthodes les plus répandues sont:

1 - La méthode d'Huder \& Amberg (1970) dans laquelle le chemin suivi permet de s'affranchir d'un certain nombre d'incertitudes liées par exemple au prélèvement du sol; cette méthode est relativement délicate à appliquer car elle nécessite l'extrapolation de la courbe de chargement.

2 - La méthode de gonflement à volume constant, selon la norme ASTM D 4546-90: la tendance de l'échantillon à gonfler est neutralisée par l'application d'une charge croissante dès que le déplacement du comparateur atteint $1 / 100 \mathrm{~mm}$. La valeur de la charge lorsque l'échantillon est stabilisé est la pression de gonflement (Sullivan \& Mac Lelland, 1969). L'utilisation de cette méthode peut être facilitée par l'emploi d'un capteur de force rigide ne permettant qu'une déformation négligeable de l'échantillon.

3 - La méthode de gonflement ou tassement sous charge constante, qui nécessite plusieurs échantillons identiques : chaque échantillon est soumis à une humidification sous charge constante; selon la valeur de la charge, il se produit un gonflement ou un effondrement du sol. En traçant la courbe des déformations volumiques en fonction des contraintes appliquées, on peut déterminer la contrainte correspondant à une déformation nulle, qui est la pression de gonflement. Selon Sridharan et al. (1986), cette courbe est une droite, tandis que Chu \& Mou (1973) trouvent une exponentielle et Philipponnat (1991), une droite ou une hyperbole.

4 - La méthode de gonflement libre suivi d'un rechargement : après la phase de gonflement libre sous fajble charge, l'échantillon quasi-saturé suit un chemin de chargement jusqu'à ce que son volume revienne à sa valeur initiale; la contrainte correspondante est la pression de gonflement.

Les chemins suivis dans les quatre méthodes ci-dessus sont très différents et les valeurs obtenues également. Selon les comparaisons réalisées par Sridaran et al. (1986), Edil \& Alazany (1992), Abduljauwad \& Al Sulei-mani (1993) et Guiras-Skandaji (1996), la méthode (4) donne toujours des valeurs sensiblement plus élevées que les autres, pouvant aller jusqu'au double de celles de la méthode (2). Les trois autres méthodes sont plus proches les unes des autres, avec des valeurs légèrement plus fortes pour la méthode (1). Seules les méthodes (2) et (3) ont été utilisées pour les mesures sur les sols algériens.

\section{2}

\section{Préparation des échantillons}

Les mesures ont été effectuées soit sur des échantillons intacts, soit sur des échantillons remaniés. Ces derniers ont été préparés par broyage puis par compactage quasi statique à vitesse lente $(1 \mathrm{~mm} / \mathrm{mn})$ de la poudre à la teneur en eau fixée (identique à celle des mesures : $w_{\text {mes }}$ ), dans un moule de 20 mm de diamètre. Les poids volumiques secs des échantillons étaient respectivement de $18 \mathrm{kN} / \mathrm{m}^{3}$ pour l'argile d'Ould Khodeir et $14,5 \mathrm{kN} / \mathrm{m}^{3}$ pour celle de Mers El Kebir. Les essais sur l'argile d'In Amenas ont été faits sur une pâte à $20 \%$ de teneur en eau.

Dans le cas des matériaux intacts, les échantillons ont été préalablement séchés à l'air libre pendant quelques jours de manière à obtenir une teneur en eau plus faible que la limite de retrait. Les teneurs en eau initiales après séchage des échantillons intacts et les teneurs en eau de compactage des échantillons remaniés, auxquelles ont été faites les mesures de gonflement libre et de pression de gonflement ( $\left.\mathrm{w}_{\text {mes }}\right)$, sont indiquées dans le tableau III. Pour les essais standards, les dimensions des échantillons sont de $70 \mathrm{~mm}$ de diamètre et $12 \mathrm{~mm}$ de hauteur. Quelques essais ont été faits avec des échantillons de 10 ou $20 \mathrm{~mm}$ de hauteur afin de regarder l'influence de ce paramètre. L'humidification des échantillons se faisait par les deux faces.

\section{3}

\section{Résultats obtenus}

Les résultats des mesures de gonflement libre à l'eau et de pression de gonflement sont indiqués dans le tableau III, où sont rappelées les valeurs des principaux paramètres d'identification.

Si l'on se réfère aux mesures de gonflement libre, on constate que les valeurs les plus élevées correspondent aux échantillons intacts d'argile de Sidi Chami et d'In Amenas (d'après Ameur, 1989), tandis que l'on obtient des valeurs fortes pour Mers El Kebir et plus faibles pour Ouled Khodeir et Abadla. Les résultats ont été reportés sous forme d'étiquettes sur les points expérimentaux correspondants, dans les graphiques de la figure 3 : ces mesures sont parfaitement cohérentes avec les différentes prévisions présentées dans le paragraphe précédent. Notons que, dans le cas d'Ouled Khodeir, la teneur en eau à laquelle a été fait l'essai est légèrement supérieure à la limite de retrait, ce qui a pu limiter le gonflement mesuré; en outre, les fortes valeurs de gonflement obtenues pour les échantillons du sondage S2 de Sidi Chami s'expliquent par la plasticité des matériaux, plus forte que la moyenne de ce sondage. Dans le cas du matériau de Mers El Kebir, le gonflement ne semble pas stabilisé au bout de 20 heures, et il aurait fallu poursuivre les essais sur une durée plus longue; les valeurs indiquées sont donc des valeurs minimales, et les valeurs de $t_{100}$ ne sont pas significatives.

En revanche, les mesures de pression de gonflement apparaissent moins cohérentes entre elles, ou si on les compare aux mesures de gonflement libre. C'est surtout le cas des essais sous charges imposées du matériau de Sidi Chami (sondage S6) pour lequel certaines valeurs de pression mesurées sont anormalement faibles. Ce point n'a pas été expliqué et pourrait résulter d'un artefact expérimental tel qu'un mauvais contrôle des conditions initiales de séchage ou de la déformation des échantillons. Pour les autres matériaux, les valeurs obtenues sont plus satisfaisantes, même s'il semble que les mesures de ce type soit moins discriminantes que les mesures de gonflement libre. 

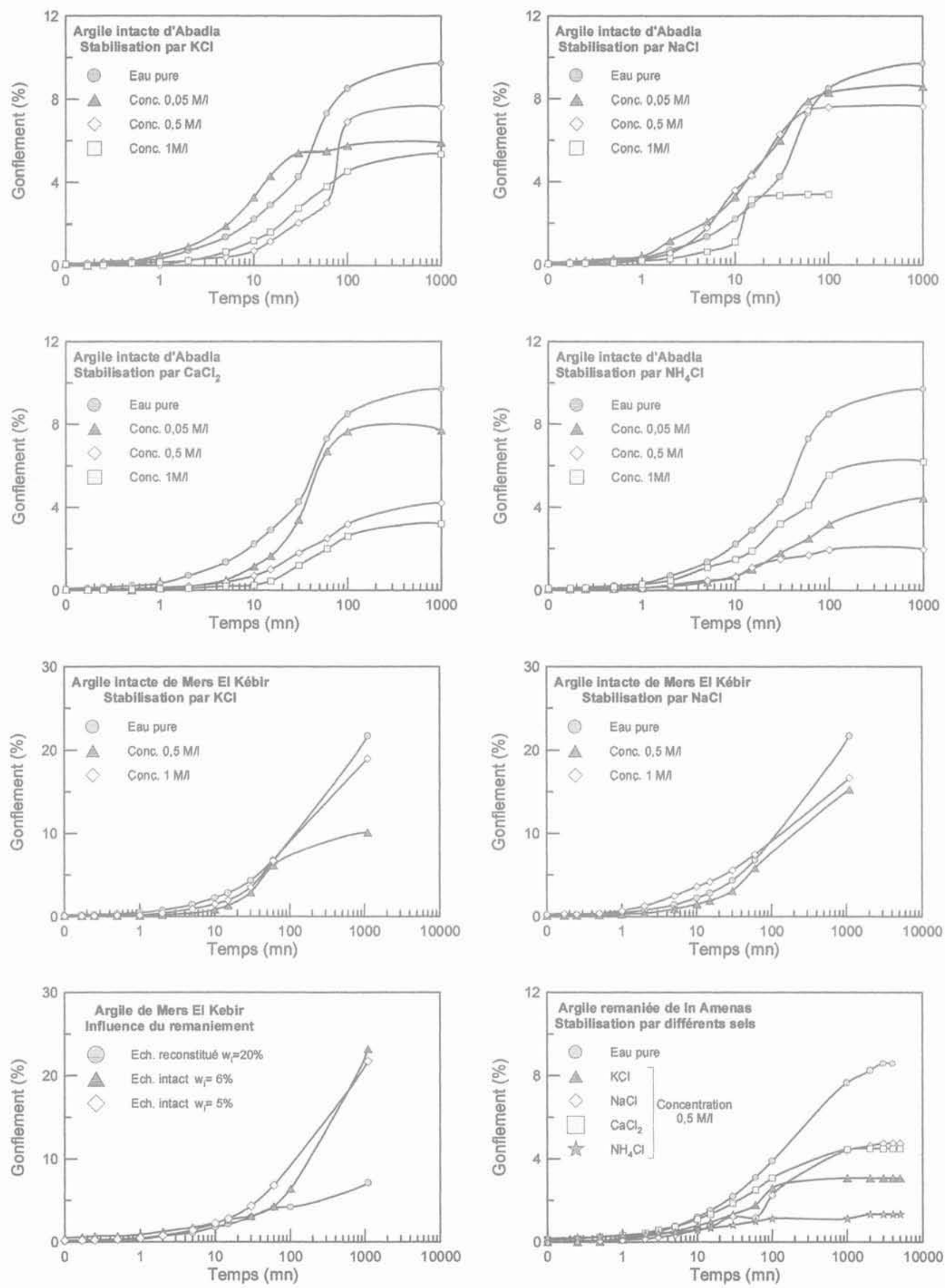

FIG. 4 Courbes de gonflement des argiles d'Abadla, Mers El Kebir et In Amenas (intactes et remaniées) en présence de différents sels.

Swelling curves of (unremolded and remolded) Abadla, Mers El Kebir and In Amenas clays with and without salts. 

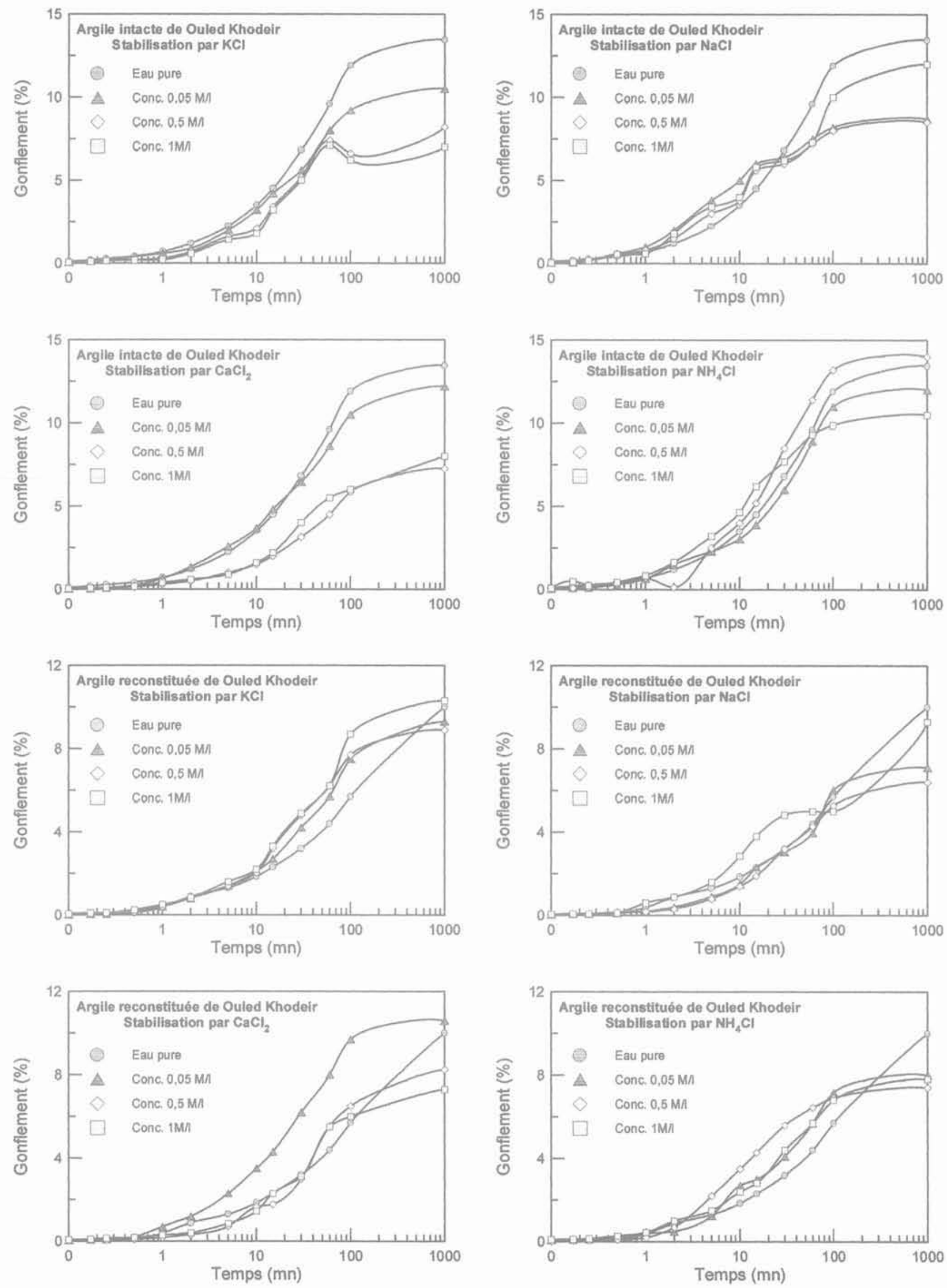

FG.5 Courbes de gonflement de l'argile d'Ouled Khodeir intacte ou remaniée en présence de différents sels. Swelling curves of unremolded and remolded Ouled Khodeir clay with and without salts, 

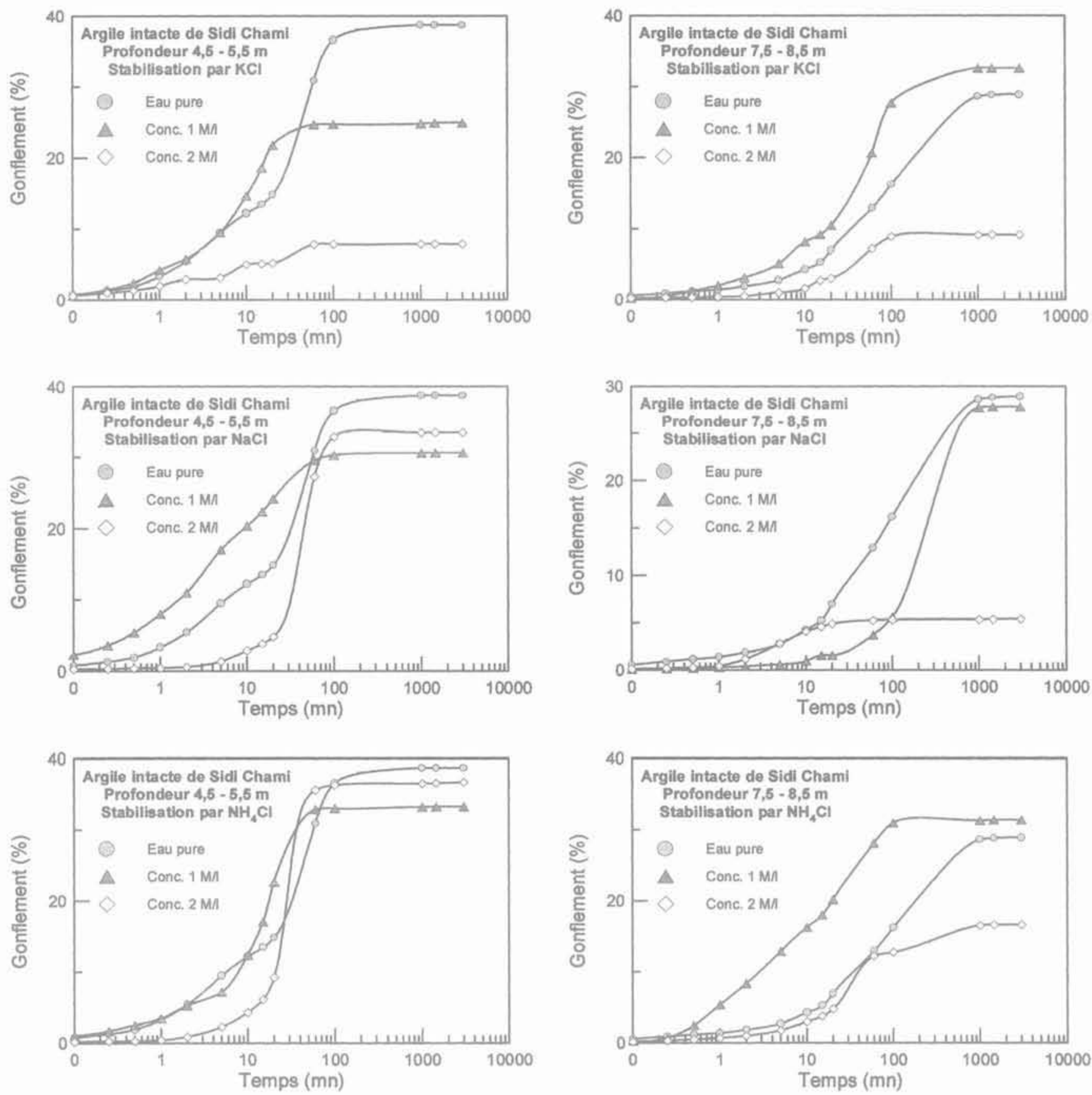

FiG.6 Courbes de gonflement de l'argile de Sidi Chami intacte à deux niveaux différents en présence de sels. Swelling curves of unremolded Sidi Chami clay at two different levels with and without salts.

Quelques mesures de gonflement libre (hors moule) ont également été faites sur les différents matériaux. qui ont montré que le phénomène était sensiblement isotrope (anisotropie de gonflement inférieure à 2).

\section{4}

\section{Influence de différents facteurs}

\section{sur la mesure du gonflement}

\section{ou de la pression de gonflement}

L'étude a porté sur le rôle de trois paramètres: la teneur en eau initiale, la hauteur de l'échantillon, et le remaniement du sol.

\section{4 .1}

Influence de la hauteur des échantillons

Les résultats des essais sur les argiles intactes de Abadla et Mers El Kebir sont indiqués dans le tableau IV. On remarque une diminution du gonflement libre mesuré lorsque la hauteur des échantillons augmente, surtout entre 12 et $20 \mathrm{~mm}$; cette diminution peut être due à l'augmentation des frottements inévitables entre l'échantillon et le moule œdométrique. Notons qu'il s'agit d'échantillons intacts présentant une dispersion importante de leurs caractéristiques, de sorte que de faibles variations des valeurs mesurées ne sont pas significatives. 
TABLEAU III Gonflements libres et pressions de gonflement mesurés sur des échantillons.

\begin{tabular}{|c|c|c|c|c|c|c|c|c|c|}
\hline Matériau & Sond. & Éch. & $W_{1}$ & It & $\begin{array}{c}W_{s} \\
\%\end{array}$ & $W_{\%}$ & $\begin{array}{l}G \\
\%\end{array}$ & $\begin{array}{l}P_{0}(1) \\
\mathrm{KPa}\end{array}$ & $\begin{array}{l}P_{1}(2) \\
k P a\end{array}$ \\
\hline Abadla & S1 & A & 40,2 & 17,1 & 14,8 & 14 & 9,7 & 100 & \\
\hline \multirow[t]{2}{*}{ Ouled Khodeir } & \multirow[t]{2}{*}{ S1 } & $\underset{\text { (intact) }}{\mathrm{A}}$ & 50 & 22,3 & 16,3 & $\begin{array}{l}18 \\
22 \\
24,9\end{array}$ & $\begin{array}{c}13,5 \\
10 \\
9,8\end{array}$ & 100 & \\
\hline & & B (rem.) & 50 & 22,3 & 16,3 & 20 & $>10$ & & \\
\hline \multirow[t]{2}{*}{ Prof. $4,5-5,5 \mathrm{~m}$} & S1 & 4 & & & & & 19.5 & 100 & \\
\hline & $\$ 2$ & $\begin{array}{l}4 \\
5\end{array}$ & 82,5 & 57,6 & & 23.5 & $\begin{array}{l}38,7 \\
60\end{array}$ & & 252 \\
\hline Sidi Chami & S3 & 4 & 105 & 71 & & & 20,4 & 200 & \\
\hline Prof. $7,5-8,5 \mathrm{~m}$ & S6 & $\begin{array}{l}1 \\
3 \\
5 \\
6\end{array}$ & $\begin{array}{l}81,5 \\
68,6 \\
69,8 \\
73,5\end{array}$ & $\begin{array}{l}62,5 \\
48,5 \\
44,2 \\
50,3\end{array}$ & & $\begin{array}{c}9,3 \\
15 \\
11.7 \\
3,3\end{array}$ & $\begin{array}{l}31,9 \\
19 \\
25,2 \\
28,9\end{array}$ & & $\begin{array}{r}150 \\
46 \\
80 \\
84\end{array}$ \\
\hline \multirow[t]{2}{*}{ Mers El Kebir } & \multirow[t]{2}{*}{ S8 } & $\begin{array}{c}\mathrm{A} \\
\text { (intace) }\end{array}$ & 52 & 27,3 & 14,7 & $5 / 6$ & $>22$ & & 310 \\
\hline & & B (rem.) & 52 & 27,3 & 14,7 & 20 & $>7,1$ ? & & \\
\hline \multirow[t]{2}{*}{ In Amenas } & S2 & $\begin{array}{c}\mathrm{A}(3) \\
\text { (intact) }\end{array}$ & 62 & 40,6 & & 4 & 30 & 3000 & \\
\hline & S1 & B (rem.) & 62,3 & 40,6 & 12 & 20 & 9,3 & 500 & \\
\hline
\end{tabular}

(1) Pression de gonflement à déformation nulle imposée.

(2) Pression de gonflement à charge imposée.

(3) D'après Ameur (1989).

TABLEAU IV Influence de la hauteur des échantillons sur le gonflement libre.

\begin{tabular}{l|c|c|c}
\multirow{2}{*}{ Matériau } & \multicolumn{3}{|c}{$\begin{array}{c}\text { Gonflement libre } \\
\text { pour des échantillons de hauteur: }\end{array}$} \\
\cline { 2 - 4 } & $10 \mathrm{~mm}$ & $12 \mathrm{~mm}$ & $20 \mathrm{~mm}$ \\
\hline Argile d'Abadla & $9,9 \%$ & $9,7 \%$ & $6,0 \%$ \\
\hline $\begin{array}{l}\text { Argile de } \\
\text { Mers El Kebir }\end{array}$ & $23,2 \%$ & $21,8 \%$ & $17,0 \%$ \\
\hline
\end{tabular}

\section{4 .2}

\section{Influence de la teneur en eau initiale des échantillons}

Dans le cas des argiles intactes de Ouled Khodeir et Mers El Kebir, des mesures de gonflement libre ont été effectuées à partir de différentes teneurs en eau initiales. Les résultats figurent dans le tableau V et montrent une tendance classique à la diminution du gonflement lorsque la teneur en eau initiale augmente (Guiras-Skandaji, 1996).

Comme nous l'avons indiqué dans l'introduction, l'augmentation de la teneur en eau initiale ne conduit à une diminution du gonflement qu'au-dessus de la limite de retrait; pour les teneurs en eau plus faibles que $w_{s 1}$, le gonflement libre ne change pas. C'est ce que l'on vérifie approximativement dans les résultats obtenus, en tenant compte de la dispersion des valeurs mesurées due à l'hétérogénéité des échantillons intacts.
TABLEAUY Influence de la teneur en eau initiale des échantillons sur le gonflement libre.

\begin{tabular}{|c|c|c|c|c|c|c|c|}
\hline Materiau & Sond. & Éch. & $W_{1}$ & $I_{p}$ & $\begin{array}{c}W_{\text {s. }} \\
\%\end{array}$ & $\begin{array}{c}W_{\text {mis }} \\
\%\end{array}$ & $\begin{array}{l}\mathrm{G} \\
\%\end{array}$ \\
\hline $\begin{array}{l}\text { Ouled } \\
\text { Khodeir }\end{array}$ & S1 & $\underset{\text { (intact) }}{\mathrm{A}}$ & 50 & 22,3 & 16,3 & $\begin{array}{l}18 \\
22 \\
24,9\end{array}$ & $\begin{array}{c}13,5 \\
10 \\
9,8\end{array}$ \\
\hline $\begin{array}{l}\text { Mers El } \\
\text { Kébir }\end{array}$ & S8 & $\begin{array}{c}\mathrm{a} \\
\text { (intact) }\end{array}$ & 52 & 27,3 & 14,7 & $\begin{array}{r}5 \\
6 \\
8 \\
10\end{array}$ & $\begin{array}{l}21,8 \\
16,8 \\
14,5 \\
14,3\end{array}$ \\
\hline
\end{tabular}

\section{4 .3}

\section{Influence du remaniement des échantillons}

La comparaison entre le gonflement libre des échantillons intacts et celui des échantillons remaniés préparés par compactage quasi statique a été faite sur trois sols : ceux d'Oled Khodeir, Mers El Kebir et In Amenas (en utilisant dans ce dernier cas les résultats de Ameur, 1989). Les valeurs obtenues sont présentées dans le tableau VI et quelques courbes sur la figure 4 . On note que, pour les trois argiles étudiées, les échantillons reconstitués ont un gonflement final plus faible que celui des échantillons intacts. Il faut toutefois remarquer que les teneurs en eau initiales des échantillons remaniés sont, dans l'ensemble, plus élevées que celles des échantillons intacts et situées au-dessus des limites de retrait correspondantes, de sorte que leur rôle ne peut être négligé, 
TABLEAUVI Influence du remaniement sur le gonflement libre.

\begin{tabular}{|c|c|c|c|c|c|c|c|}
\hline \multirow[t]{2}{*}{ Matériau } & \multirow[t]{2}{*}{$W_{L}$} & \multirow[t]{2}{*}{$I_{p}$} & \multirow{2}{*}{$\frac{W_{S I}}{\%}$} & \multicolumn{2}{|c|}{ Ech intacts } & \multicolumn{2}{|c|}{ Éch. remaniés } \\
\hline & & & & $\begin{array}{c}W_{\text {mex }} \\
\%\end{array}$ & $\begin{array}{l}G \\
\%\end{array}$ & $\begin{array}{c}\mathrm{W}_{\text {matr. }} \\
\%\end{array}$ & $\begin{array}{l}G \\
\%\end{array}$ \\
\hline $\begin{array}{l}\text { Ouled } \\
\text { Khodeir }\end{array}$ & 50 & 22,3 & 16,3 & 18 & 13,5 & 20 & $>10$ \\
\hline $\begin{array}{l}\text { Mers El } \\
\text { Kébir }\end{array}$ & 52 & 27,3 & 14,7 & $5 / 6$ & $>22$ & 20 & $\geq 7,1$ ? \\
\hline In Amenas & 62,3 & 40,6 & 12 & 4 & $30(1)$ & 20 & 9,3 \\
\hline
\end{tabular}

(1) D'après Ameur (1989).

\section{7}

\section{Stabilisation chimique du gonflement par des sels}

De nombreux travaux ont montré que des sels minéraux tels que les chlorures de calcium ou de potassium, ou le carbonate de potassium, pouvaient réduire sensiblement le gonflement des argiles naturelles. L'efficacité d'un sel peut être analysée, en première approximation, à partir du concept d'، épaisseur de la double-couche diffuse ») (Komine \& Ogata, 1992). En effet, le gonflement est souvent considéré comme étant directement lié à l'augmentation d'épaisseur de la couche diffuse, même si certaines recherches plus récentes (Qi et al., 1996) ont montré qu'il ne s'agissait que d'un aspect très partiel du phénomène. Nous avons indiqué dans le tableau VII les épaisseurs de doublecouches calculées de façon classique (Mitchell, 1976), pour différents sels et différentes concentrations. On peut ainsi classer a priori l'efficacité des traitements par ordre croissant :

$$
\begin{gathered}
+(0,05 \mathrm{M})<+(0,05 \mathrm{M})<+(0,5 \mathrm{M})<+(1 \mathrm{M})<+(2 \mathrm{M}) \\
\text { ou }{ }^{++}(0,5 \mathrm{M})<+(1 \mathrm{M})
\end{gathered}
$$

oú + et +* représentent la valence du cation, et la quantité entre parenthèses, la molalité du sel. Il faut également tenir compte des possibilités d'hydratation de chaque cation, fonction de sa charge électrique et de son rayon, qui conduit, dans le cas des trois cations monovalents, au classement suivant d'efficacité croissante :

$$
\mathrm{Na}<\mathrm{K}<\mathrm{NH}_{4}
$$

Enfin, la nature des cations et leur taille peuvent jouer un rôle majeur dans les substitutions ioniques qui se produisent à l'intérieur des feuillets argileux. On sait que l'échange des ions sodium par des ions calcium au sein d'une montmorillonite, par exemple, conduit à un matériau moins gonflant.

\section{Méthode utilisée}

Pour étudier l'effet des sels sur le gonflement libre des sols étudiés, les échantillons ont été saturés dans une cellule cedométrique par des solutions salines à différentes concentrations. Ensuite le gonflement a été mesuré en fonction du temps jusqu'à stabilisation. Le gonflement final est comparé au gonflement final de l'argile saturée à l'eau. La réduction du gonflement final exprimée en pourcents est la différence entre les gonflements à l'eau et avec les solutions salines, rapportée au gonflement final en présence d'eau.

Quatre sels ont été utilisés :

- le chlorure de potassium $\mathrm{KCl}$;

- le chlorure de sodium $\mathrm{NaCl}$;

- le chlorure de calcium $\mathrm{CaCl}_{2}$;

- le chlorure d'ammonium $\mathrm{NH}_{4} \mathrm{Cl}$.

Les molalités des solutions salines sont de 0,05 mole/litre $(\mathrm{M}) ; 0,5 \mathrm{M} ; 1 \mathrm{M}$ et $2 \mathrm{M}$.

\section{2}

\section{Résultats obtenus}

Les courbes de gonflement libre en fonction du logarithme du temps pour diverses solutions salines et différentes concentrations sont représentées sur les figures 4 à 6 . Les valeurs de gonflement final et de réduction du gonflement sont résumées dans le tableau VIII, où sont également reportées les valeurs de $t_{100}$, temps correspondant à la fin du gonflement primaire, comme indicateur de la cinétique des phénomènes (tous les échantillons ayant sensiblement la même hauteur et les mêmes conditions de drainage).

11 est difficile de tirer une conclusion globale de l'action des différents sels car, si l'on constate bien en général un effet positif sur la réduction du gonflement, cet effet varie fortement d'une argile à l'autre. La dispersion des résultats est en partie due à l'hétérogénéité des matériaux étudiés du fait que les mesures de gonflement se font toutes sur des échantillons différents.

Dans la majorité des cas, il semble que des réductions substantielles soient obtenues avec de fortes concentrations en sels. C'est notamment le cas pour l'argile de Sidi Chami, la plus gonflante, où les réductions atteignent $80 \%$. Si l'on considère les différents sels utilisés, on constate que le chlorure de calcium et, à un moindre degré, le chlorure d'ammonium, à une concentration de 0,5 à $1 \mathrm{M} / 1$, conduisent presque toujours à de bons résultats, même si ceux-ci ne sont pas nécessairement optimum.

Les observations précédentes sont en accord avec l'échelle théorique d'efficacité présentée précédem-

\begin{tabular}{|c|c|c|c|c|c|c|c|c|}
\hline \multirow[b]{2}{*}{ Concentration en $\mathrm{M} / 1$} & \multicolumn{5}{|c|}{$\begin{array}{l}\text { Cations monovalents } \\
\text { Sels: KCl, NaCl, } \mathrm{NH}_{4} \mathrm{Cl}\end{array}$} & \multicolumn{3}{|c|}{$\begin{array}{l}\text { Cations divalents } \\
\text { Sel: } \mathrm{CaCl}_{2}\end{array}$} \\
\hline & $\begin{array}{c}10^{-3} \\
\text { (eau pure) }\end{array}$ & 0,05 & 0,5 & 1 & 2 & 0,05 & 0,5 & 1 \\
\hline Épaisseur de la double-couche (nm) & 9,5 & 1,35 & 0,43 & 0,30 & 0,20 & 0,68 & 0,21 & 0,15 \\
\hline
\end{tabular}

TABLEAUVII Épaisseur de la double-couche diffuse pour différents sels à différentes concentrations (d'après Mitchell, 1976). 
TABLEAU VIII Gonflements libres et variations relatives de volume mesurés en présence de différentes solutions salines à plusieurs concentrations.

\begin{tabular}{|c|c|c|c|c|c|c|c|c|c|c|c|c|c|c|c|c|c|}
\hline & & \multirow[t]{2}{*}{$\mathrm{H}_{2} \mathrm{O}$} & \multicolumn{4}{|c|}{$\mathrm{KCl}$ tCone. en $\mathrm{M} / 1$} & \multicolumn{4}{|c|}{$\mathrm{NaCl}$ (Conc. en $\mathrm{M} /$ ) } & \multicolumn{3}{|c|}{$\mathrm{CaCl}_{2}$ (Conc. en $\mathrm{M} /$ ) } & \multicolumn{4}{|c|}{$\mathrm{NH}_{+} \mathrm{Cl}$ (Conc. en $\mathrm{M} / \mathrm{I}$} \\
\hline & & & 0,05 & 0,5 & 1 & 2 & 0,05 & 0,5 & 1 & 2 & 0,05 & 0,5 & 1 & 0,05 & 0,5 & 1 & 2 \\
\hline $\begin{array}{l}\text { Abadla } \\
\text { S1 intacte }\end{array}$ & $\begin{array}{c}G(\%) \\
\Delta G / G_{H / O} \\
t_{100}(m n)\end{array}$ & $\begin{array}{l}9,7 \\
82\end{array}$ & $\begin{array}{c}5,9 \\
39 \% \\
67\end{array}$ & $\begin{array}{c}7,6 \\
22 \% \\
2800\end{array}$ & $\begin{array}{c}5,4 \\
44 \% \\
250\end{array}$ & & $\begin{array}{c}8,6 \\
11 \% \\
81\end{array}$ & $\begin{array}{c}7.7 \\
21 \% \\
81\end{array}$ & $\begin{array}{c}3.4 \\
65 \% \\
61\end{array}$ & & $\begin{array}{c}7,7 \\
21 \% \\
88\end{array}$ & $\begin{array}{c}4,2 \\
57 \% \\
535\end{array}$ & $\begin{array}{l}3,2 \\
67 \% \\
655\end{array}$ & $\begin{array}{c}4,5 \\
54 \% \\
520\end{array}$ & $\begin{array}{c}2 \\
79 \% \\
220\end{array}$ & $\begin{array}{c}6,2 \\
36 \% \\
535\end{array}$ & \\
\hline $\begin{array}{l}\text { S1rintacte } \\
\text { Ouled Khodeir }\end{array}$ & $\begin{array}{c}G(\%) \\
\Delta G / G_{1 / 2} \\
t_{100}(m n)\end{array}$ & $\begin{array}{l}13,5 \\
235\end{array}$ & $\begin{array}{c}10,5 \\
22 \% \\
340\end{array}$ & $\begin{array}{c}8,2 \\
39 \% \\
265\end{array}$ & $\begin{array}{c}7 \\
48 \% \\
385\end{array}$ & & $\begin{array}{c}8,7 \\
36 \% \\
235\end{array}$ & $\begin{array}{c}8,5 \\
37 \% \\
160\end{array}$ & $\begin{array}{c}12 \\
11 \% \\
340\end{array}$ & & $\begin{array}{l}12,2 \\
10 \% \\
304\end{array}$ & $\begin{array}{c}7,3 \\
46 \% \\
334\end{array}$ & $\begin{array}{c}8 \\
41 \% \\
400\end{array}$ & $\begin{array}{c}12 \\
11 \% \\
250\end{array}$ & $\begin{array}{c}14 \\
-3 \% \\
190\end{array}$ & $\begin{array}{c}10,5 \\
22 \% \\
70\end{array}$ & \\
\hline reconstituée & $\begin{array}{c}G(\%) \\
\left.\Delta G / G_{H y}\right) \\
t_{100}(m n)\end{array}$ & $\begin{array}{c}>10 \\
>1000\end{array}$ & $\begin{array}{l}9,3 \\
7 \% \\
130\end{array}$ & $\begin{array}{c}8,9 \\
11 \% \\
130\end{array}$ & $\begin{array}{c}10,3 \\
-3 \% \\
110\end{array}$ & & $\begin{array}{c}7,1 \\
29 \% \\
100\end{array}$ & $\begin{array}{c}6.4 \\
36 \% \\
170\end{array}$ & $\begin{array}{c}>9,3 \\
7 \% \\
>1000\end{array}$ & & $\begin{array}{c}10,6 \\
-6 \% \\
110\end{array}$ & $\begin{array}{c}8,3 \\
17 \% \\
100\end{array}$ & $\begin{array}{c}7,3 \\
27 \% \\
85\end{array}$ & $\begin{array}{c}8 \\
20 \% \\
110\end{array}$ & $\begin{array}{c}7,4 \\
26 \% \\
60\end{array}$ & $\begin{array}{c}7,8 \\
22 \% \\
110\end{array}$ & \\
\hline $\begin{array}{l}\text { S2: Prof, } 4,5 \cdot 5,5 \mathrm{~m} \\
\text { intacte } \\
\text { Sidi Chami }\end{array}$ & $\begin{array}{c}G(\%) \\
\left.\Delta G / G_{B y}\right) \\
t_{m,}(m n)\end{array}$ & $\begin{array}{l}38,7 \\
700\end{array}$ & & & $\begin{array}{c}24,9 \\
36 \% \\
53\end{array}$ & $\begin{array}{c}7,9 \\
80 \% \\
80\end{array}$ & & & $\begin{array}{c}30,6 \\
21 \% \\
80\end{array}$ & $\begin{array}{c}33.5 \\
13 \% \\
90\end{array}$ & & & & & & $\begin{array}{c}33,3 \\
14 \% \\
80\end{array}$ & $\begin{array}{l}36,7 \\
5 \% \\
80\end{array}$ \\
\hline $\begin{array}{l}\text { S6: Prof. } 7,5-8,5 \mathrm{~m} \\
\text { intacte }\end{array}$ & $\begin{array}{c}G(\%) \\
\Delta G / G_{H \% O} \\
t_{100}(m n)\end{array}$ & $\begin{array}{l}28,9 \\
700\end{array}$ & & & $\begin{array}{c}32.6 \\
-13 \% \\
110\end{array}$ & $\begin{array}{c}9,2 \\
68 \% \\
90\end{array}$ & & & $\begin{array}{l}27,8 \\
4 \% \\
630\end{array}$ & $\begin{array}{c}5,4 \\
81 \% \\
20\end{array}$ & & & & & & $\begin{array}{c}31,4 \\
-9 \% \\
80\end{array}$ & $\begin{array}{c}16,6 \\
43 \% \\
80\end{array}$ \\
\hline $\begin{array}{l}\text { Mers El Kebir } \\
\text { SB: intacte }\end{array}$ & $\begin{array}{c}G(\%) \\
\Delta G / G_{\mathrm{H}_{2}} \\
t_{100}(\mathrm{mrn})\end{array}$ & $\begin{array}{l}>22 \\
>1000\end{array}$ & & $\begin{array}{c}10.1 \\
54 \% \\
606\end{array}$ & $\begin{array}{l}>19 \\
13 \% \\
892\end{array}$ & & & $\begin{array}{c}>15,3 \\
30 \% \\
>1000\end{array}$ & $\begin{array}{c}>16,7 \\
23 \% \\
>1000\end{array}$ & & & & & & & & \\
\hline In Amenas & $\begin{array}{c}G(\%) \\
\Delta G / G_{\% \%} \\
t_{100}(m n)\end{array}$ & $\begin{array}{c}9,3 \\
2428\end{array}$ & & $\begin{array}{c}3.1 \\
67 \% \\
614\end{array}$ & & & & $\begin{array}{l}4.8 \\
48 \% \\
1000\end{array}$ & & & & $\begin{array}{l}4,5 \\
52 \% \\
457\end{array}$ & & & $\begin{array}{l}1,3 \\
86 \% \\
300\end{array}$ & & \\
\hline
\end{tabular}

ment. Cependant, on note aussi, de façon plus surprenante, qu'avec l'argile d'Abadla ou celle de Sidi Chami, le chlorure de sodium conduit à des réductions importantes du gonflement.

La nature minéralogique de l'argile joue également un rôle essentiel, comme on pouvait s'y attendre : alors que l'efficacité des différents sels reste médiocre dans le cas des argiles de Ouled Khodeir ou Mers El Kebir, pour lesquelles les réductions de gonflement ne dépassent guère $50 \%$, tous les sels semblent efficaces dans le cas de l'argile d'In Amenas (particulièrement $\mathrm{NH}_{4} \mathrm{Cl}$ et $\mathrm{KCl}$.

Dans quelques cas très rares, la présence de sels conduit à une augmentation du gonflement libre, notamment avec l'argile de Sidi Chami. Ces augmentations restent très faibles et dans les limites des incertitudes liées à la dispersion des échantillons.

Du point de vue de la cinétique du gonflement qui peut être déduite des valeurs des t courbes sur les figures 4 à 6 , on remarque que la présence des sels se traduit, le plus souvent, soit par un ralentissement du gonflement des matériaux intacts, soit à défaut par la même cinétique. Dans les deux cas d'argiles remaniées, en revanche, on note le phénomène inverse, c'est-à-dire une accélération du gonflement en présence de sels. Il faut néanmoins tempérer ces conclusions par le fait que les déterminations des $t_{100}$ sont parfois difficiles à faire dans le cas des argiles traitées en raison de la forme peu régulière de certaines courbes de gonflement qui paraissent progresser par bonds successifs ; en outre, pour certains essais (argiles de Mers El Kebir et Ouled Khodeir, notamment), la durée des mesures (1 000 minutes ou plus) apparaît trop limitée pour atteindre l'équilibre en échelle logarithmique.

L'application de la technique de stabilisation des sols gonflants par des sels à des ouvrages réels doit être envisagée avec prudence, notamment dans le cas oủ les essais de laboratoire ne mettent pas en évidence de façon claire l'obtention d'un équilibre. Il n'est pas impossible alors que le gonflement des matériaux traités puisse rejoindre celui des matériaux non traités à l'échelle de la vie d'un ouvrage; pour éclaircir ce point. il faudrait mener des études très longues, si possible dans les conditions du site. Par ailleurs, la possibilité de lixiviation des sels à long terme par les eaux de pluie ne peut pas être écartée.

\section{8}

\section{Conclusion}

Les études qui ont été menées (minéralogie, essais d'ídentification et essais de gonflement) ont confirmé le caractère gonflant des cinq sols étudiés. Néanmoins, les deux argiles provenant du sud-ouest algérien ont un potentiel de gonflement moins élevé que les sols provenant des autres régions. Ceci n'écarte pas la menace que constituent ces sols pour les constructions légères, comme il a été observé sur les sites où plusieurs habitations à rez-de-chaussée ont subi des dégradations importantes telles que des fissures en escalier ou inclinés à $45^{\circ}$.

La comparaison de cinq méthodes différentes de prévision du potentiel de gonflement à partir des paramètres d'identification a permis d'établir un classement cohérent (au moins d'un point de vue qualitatif) des risques de gonflement des sols: ce classement a été confirmé par les mesures directes de gonflement libre et de pression de gonflement réalisées.

Des précautions doivent être prises pour protéger ces sols des infiltrations d'eau afin d'éviter que le gonflement puisse se produire sous les ouvrages, ou bien procéder à la stabilisation des sols avant les travaux de 
fondations, par exemple en injectant des solutions stabilisantes telles que les solutions salines qui ont montré leur efficacité pour réduire le gonflement pour un coût limité. Il ressort néanmoins des essais réalisés qu'il est indispensable d'effectuer une étude spécifique avant chaque cas de traitement envisagé afin de définir la nature et la concentration des solutions salines à utiliser sur un site donné; cette étude préalable doit aussi prendre en compte la stabilité à long terme du traitement, c'est-à-dire les risques de lixiviation des sels. L'utilisation de surfactants permet également un traitement efficace des sols argileux (Fleureau et al., 1988).

\section{Bibliographie}

Ameur B. - "Sols gonflants : étude de cas Comptes rendus du Symp. Int. de Mécanique des Sols de Tiaret (Algérie), juin 1989, CORT-LTPO (éd.), tome 1, 1989.

Abduljauwad S.N., Al Suleimani G. a Determination of swell potential of A Qatif clay n, Geotechnical Testing J. vol. 16, n4, 1993, p. 469-484

Biarez J., Fleureau J.M., Zerhouni M.I. Soepandji B.S. - « Variations de volume des sols argileux lors de cycles de drainage-humidification $\gg$. Revue Française de Géotechnique, 41, 1988, p. 63-71.

Brackley I.J.A. - $\alpha$ A model of unsaturated clay structure and its application to swell behaviour n. Proc. 6th Reg Conf. for Africa on Soil Mech. and Found Eng., Durban, vol. 1, 1975, p. 65-79.

Chen F.H. - u Foundations on expansive solis x. Developments in Geotechnical Enqineering, vol. 54, Elsevier Publishing Co., Amsterdam, 1988, 464 p.

Chu T.Y., Mou C.H. - « Volume change characteristics of expansive soils determined by controlled-suction tests 1 . Proc. 3rd Int. Conf. on Expansive soils, Haifa, vol. 1. 1973, p. 177-185

Edil T.B., Alazany A.S. $-\alpha$ Lateral swelling pressures 2. Proc. 7 th Int. Conf on expansive soils, Dallas, vol. 1, 1992, p. $227-232$

Fleureau J.M. Wignyodarsono L. Zerhouni M.I. - - Effects of surfactants on the mechanical properties of a kaolinite in relation to the solid-liquid contact angles ग. Can. Geotech. J., 25, 1988 . p. 675-683.

Fleureau J.M., Kheirbek-Saoud S., Soemitro R., Taibi S, - " Behavior of clayey soils on drying-wetting paths mi. Can Geotech. $J, 30, n^{\circ} 2,1993$, p. 287-296.
Fleureau J.M., Kheirbek-Saoud S. Imbert C. - " Mechanical behaviour of an expansive clay on drying-wetting paths x. Proc. 3rd Int. Congress on Environmental Geotechnics, Lisboa, September 7-11th, Balkema (ed.), Rotterdam, vol. 1, 1998, p. 101-106.

Guiras-Skandaii H - « Déformabilité des sols argileux non saturés : étude expérimentale et application à la modélisation x. Thèse de Doctorat soutenue le 5 juin 1996, Institut national polytechnique de Lorraine, 1996, 315 p.

Holtz W.G., Gibbs H.J. - " Engineering properties of expansive clays $\pi$. Transactions ASCE, vol. 121, 1956, p. 641-677.

Huder J., Amberg G. - " Quellung in mergel, opalinuston and anhydrit ». Schweizerische Bauzeitung, ${ }^{\circ} 43,1970$, p. $975-980$,

Komine H, Ogata N. - $\alpha$ Swelling characteristics of compacted bentonite w. Proc. 7 th Int. Conf. on Expansive soils, Dallas, 1992, p. 216-221.

Komornik. A. David D - " Prediction of swelling pressure of clays $\pi$. J. Soil Mech. and Found. Eng. Div. ASCE, 95. SM1, 1969, p. 209-225.

Meisina C. - « Critères et méthodes pour l'évaluation du gonflement de différents types de sols et terrains de la plaine du Pô \#. Geologia Applicata ed Idrogeologia, vol. XXVIII. 1993, p. 571-579 (en italien).

Meísina C. $-\alpha$ Critères et méthodes pour l'évaluation du gonflement de différents types de sols de la région de Pavie n. Thèse de Doctorat soutenue en février 1996. Università degli Studi di Pavia, 1996, 281 p. (en italien).

Mitchell J.K. - a Fundamentals of soil behaviour $\gg$. John Wiley \& sons, New York 1976.
Philipponnat G. - « Retrait-gonflement des argiles, proposition de méthodologie n, Revue Française de Géotechnique, $n^{\circ} 57$. 1991, p. 5-22.

Qi Y.. Al-Mukhtar M. Alcover J.F. Bergaya F. - "Coupling analysis of macroscopic and microscopic behaviour in highly consolidated Na-laponite clavs 17. Applied Clay Science, 11, 1996, p. 185197.

Seed M.B., Woodward R.J., Lundgren R. " Prediction of swelling potential for compacted clays v. J. Soil Mech. and Found. Eng. Div, ASCE, 88, 1962, p. 107131.

Sridharan N., Rao A.S, Sivapullaiah P.V. "Swelling pressures of clays ". Geotechnical Testing J., vol. 9, $n^{\circ} 1,1986$, p. 24-33.

Sullivan R.A., Mac Lelland B, - « Predicting heave of buildings on unsaturated clay ». Proc. 2nd Int. Conf. on Expansive Soils. Texas A \& M University, 1989.

Tavlor R.K.. Smith T.J. - u The engineering geology of clay minerals: swelling, shrinking and mudrock breakdown 8 . Clay Minerals, 21, 1985, p. 235-260.

Tran Ngoc Lan - $\alpha$ Nouvel essai d'identification des sols: l'essai au bleu de méthylène w. Bulletin de liaison des Ponts et Chaussées, 88, mars-avril, 1977, p. 136-137.

Vijayvergiya V.N., Ghazzaly O.I. - $\alpha$ Prediction of swelling potential for natural clays 3. Proc. 3rd Int. Conf. On Expansive Soils, Haifa, vol. 1, 1973, p. 227-238.

Williams A.B., Donaldson. G.W. - $\alpha$ Developments related to building on expansive soils in South Africa : 1973-1980 $\mathrm{n}$. Proc, 4th Int. Conf. on Expansive Soils. Denver, vol. 2, 1980, p. 834-844. 